\title{
Latitude survey investigation of galactic cosmic ray solar modulation during 1994-2007
}

\author{
W. Nuntiyakul, ${ }^{a, b, c}$ P. Evenson, ${ }^{d}$ D. Ruffolo*, ${ }^{a, b}$ A. Sáiz, ${ }^{a, b}$ J. W. Bieber, ${ }^{d}$ J. Clem, ${ }^{d}$ R. \\ Pyle, ${ }^{d}$ M. L. Duldig, ${ }^{e}$ J. E. Humble ${ }^{e}$
}

${ }^{a}$ Department of Physics, Faculty of Science, Mahidol University, Bangkok 10400, Thailand.

${ }^{b}$ Thailand Center of Excellence in Physics, CHE, Ministry of Education, Bangkok 10400,

Thailand.

${ }^{c}$ Faculty of Science, Chandrakasem Rajabhat University, Bangkok 10900, Thailand.

${ }^{d}$ Bartol Research Institute, University of Delaware, Newark, DE 19716, USA.

${ }^{e}$ School of Physical Sciences, University of Tasmania, Hobart, Tasmania 7001, Australia.

E-mail: w. nuntiyakul@gmail.com, evenson@bartol.udel.edu,

david.ruf@mahidol.ac.th, alejandro.sai@mahidol.ac.th,

jwbieber@bartol.udel.edu, jmc@udel.edu, rogerpyle@gmail.com,

marc.duldig@utas.edu.au, john.humble@utas.edu.au.

The Galactic cosmic ray spectrum exhibits subtle variations over the 22 yr solar magnetic cycle in addition to the more dramatic variations over the $11 \mathrm{yr}$ sunspot cycle. Neutron monitors are large ground-based detectors that provide accurate measurements of variations in the cosmic ray flux at the top of the atmosphere above the detector. At any given location the magnetic field of the Earth excludes particles below a well-defined rigidity (momentum per unit charge) known as the cutoff rigidity, which can be accurately calculated using detailed models of the geomagnetic field. By carrying a neutron monitor to different locations, e.g., on a ship, the Earth itself serves as a magnet spectrometer. By repeating such latitude surveys with identical equipment a sensitive measurement of changes in the spectrum can be made. In this work, we analyze data from the 1994 through 2007 series of latitude surveys conducted by the Bartol Research Institute, the University of Tasmania, and the Australian Antarctic Division. We confirm the curious "crossover" in spectra measured near solar minima during epochs of opposite solar magnetic polarity, and show that it is related directly to a sudden change in the spectral behavior of solar modulation at the time of the polarity reversal, as revealed from contemporaneous variations in the survey data and a fixed station. We suggest that the spectral change and crossover result from the interaction of effects due to gradient/curvature drifts with a systematic change in the interplanetary diffusion coefficient caused by turbulent magnetic helicity.

The 34th International Cosmic Ray Conference,

30 July- 6 August, 2015

The Hague, The Netherlands

\footnotetext{
${ }^{*}$ Speaker.
} 


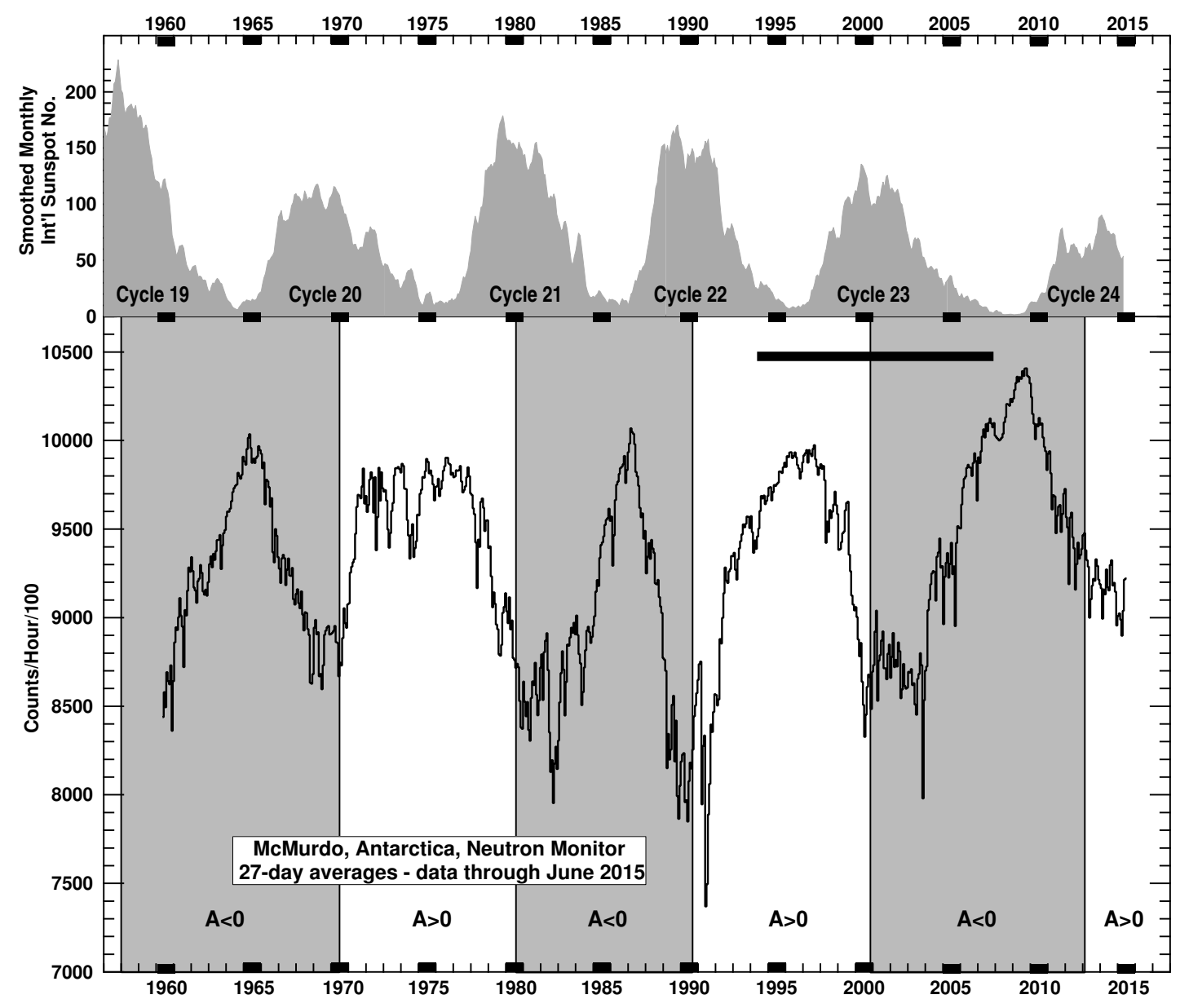

Figure 1: Smoothed monthly international sunspot number (using 5-month boxcar smoothing) and McMurdo neutron monitor count rate as a function of time. The long-term drift at McMurdo has been corrected following [20]. A neutron monitor count rate indicates the Galactic cosmic ray flux, which undergoes "solar modulation" in association with solar activity. Solar modulation includes dramatic 11-year variations with the sunspot cycle, and a 22-year variation with the solar magnetic cycle, seen here in changes in the solar modulation pattern between positive $(A>0)$ and negative $(A<0)$ magnetic polarity. In this work we present observations of spectral changes in Galactic cosmic rays in association with solar modulation and changing solar magnetic polarity for the time period 1994-2007, indicated by a horizontal bar. (Modified from [19].)

\section{Introduction}

The Galactic cosmic ray (GCR) flux in the solar system is strongly influenced by solar variations [12], a process known as solar modulation. This is dominated by the roughly 11 -year sunspot cycle in which the Galactic cosmic ray flux decreases during sunspot maximum, the time period of maximum solar activity, and increases during sunspot minimum (Figure 1).

However, there is also a roughly 22-year GCR variation corresponding to the solar magnetic cycle, wherein the dominant solar magnetic polarity reverses at each sunspot maximum [21]. In other words, 11-year periods with opposite magnetic polarity exhibit distinct GCR variations. These effects are associated with a variety of interesting phenomena, such as guiding center drifts, 
latitudinal GCR gradients, particle charge sign dependence, and changing diffusion coefficients, e.g., $[3,13,14]$. These phenomena depend on the sign of $q A$, where $q$ is the particle charge and $A$ is the solar magnetic polarity. Effects of solar magnetic polarity are clearly seen in the GCR flux as a function of time, which is more "pointy" or "flat-topped" in alternating sunspot cycles [15, 22] (see Figure 1), and by comparing fluxes of particles of the same charge to mass ratio but opposite charge sign, such as electrons to positrons or protons to antiprotons [2].

One of the most puzzling aspects of modulation phenomenology is the so-called "crossover" in spectral form during opposite magnetic polarity epochs [18]. The crossover is one manifestation of a dependence of spectral shape on solar magnetic polarity. This has been observed by means of ship-borne neutron monitor surveys that study the GCR spectrum by traveling across a wide range of geomagnetic cutoff rigidities. Reports of crossovers in the literature have usually compared observations at two different time periods "near solar minimum," but it has recently become clear that different solar minima can have different levels of solar modulation [20]. The question of whether or at what rigidity the spectra intersect at successive solar minima depends on the relative level of modulation at the two time periods. Actually, the key physical issue is whether solar modulation of GCR spectra is independent of solar magnetic polarity for similar modulation conditions. Such a polarity dependence can be demonstrated by the existence of a spectral crossover at any similar modulation conditions for different magnetic polarities, or equivalently, if a model of solar modulation that matches the GCR spectral evolution during one solar magnetic polarity systematically deviates from that for the opposite polarity.

Additional questions that immediately come to mind involve the transition in solar modulation from one polarity state to the other. Is this transition smooth or abrupt? Alternatively, the crossover might be a phenomenon that appears as a result of some special conditions that appear and disappear only very near solar minimum. To investigate the nature of this transition, a series of neutron monitor latitude surveys was conducted between 1994 and 2007 by the Bartol Research Institute of the University of Delaware, the University of Tasmania, and the Australian Antarctic Division. We present two types of results from the survey data: the classic approach of determining response functions, which confirms a crossover when comparing similar modulation conditions near successive solar minima, and an analysis of contemporaneous variations in the survey data and data from a fixed NM station, which reveals a spectral change associated with a major transition in some aspect of modulation that occurs nearly simultaneously with the solar polarity reversal. The key results have also been presented by [19].

\section{Observations and Data Reduction}

In our series of surveys, atmospheric neutrons were detected by a ship-borne mobile neutron monitor technically known as a 3NM64. NM64 refers to the basic design [5] and "3" refers to our use of three independent neutron counters. These were installed in an insulated shipping container (called the "TasVan") as shown in Figure 2. The 3NM64 mobile monitor was operated during 1994-2007 aboard one of two U.S. Coast Guard icebreakers, the Polar Sea or the Polar Star, which traversed the Pacific Ocean from Seattle, USA to McMurdo, Antarctica and back during an annual 6-month voyage. We refer to a "survey year" by the year in which the voyage began; each voyage then extended throughout the Austral Summer into the next calendar year. For example, 


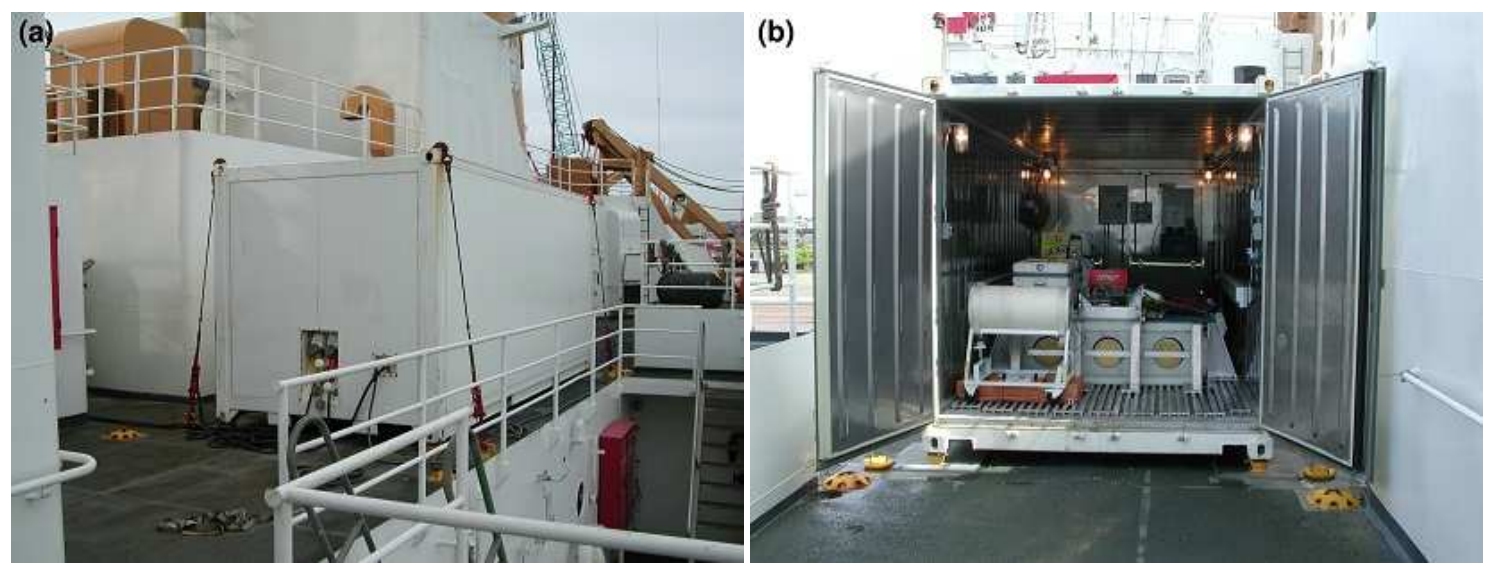

Figure 2: (a) Insulated container ("TasVan") used for 3NM64 neutron monitor latitude surveys on the Polar Star and Polar Sea. (b) 3NM64 installed inside the container. For this and several other voyages a small calibration neutron monitor [16] was installed near the door. (Figure from [19].)

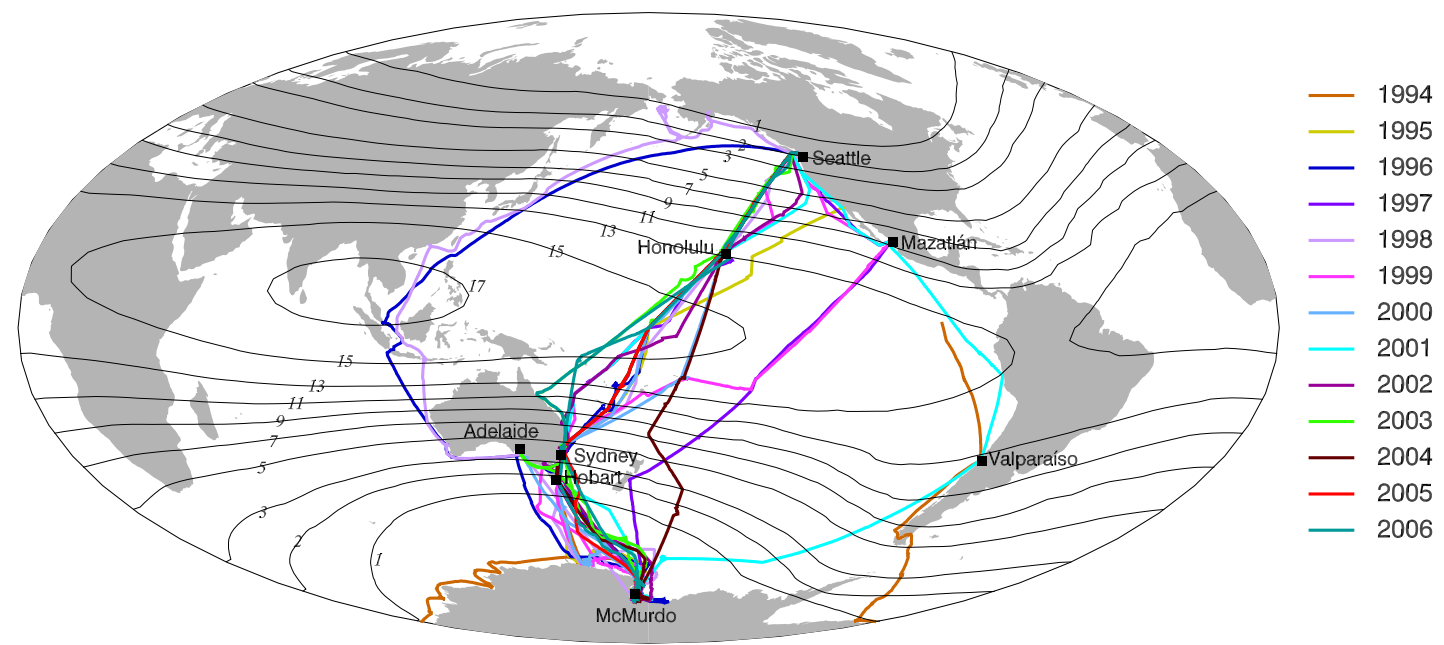

Figure 3: Tracks of the ship-borne neutron monitor latitude surveys for 1994-2007, superimposed on contours of the vertical cutoff rigidity in GV. (Figure from [19].)

data for "survey year 1997" refer to data of the voyage from October, 1997 to April, 1998. We obtained data for 13 consecutive survey years (from 1994 to 2006). The geographic routes of all surveys are plotted in Figure 3; also shown for reference are contours of constant 1980 vertical effective geomagnetic cutoff rigidity.

A ship-borne neutron monitor is not as stable as a fixed neutron monitor station. Several steps of data reduction were required to obtain clean response functions, i.e., curves of count rate vs. cutoff rigidity. First, we used the count rate ratios of individual tubes from each data interval (typically $30 \mathrm{~min}$ ) to reject data from noisy tubes and correct data from the remaining tubes to account for missing tubes. Then we plotted daily tube ratios, which have greater statistical accuracy and are much more sensitive indicators of tube drifts or noise, and anomalous time periods were 

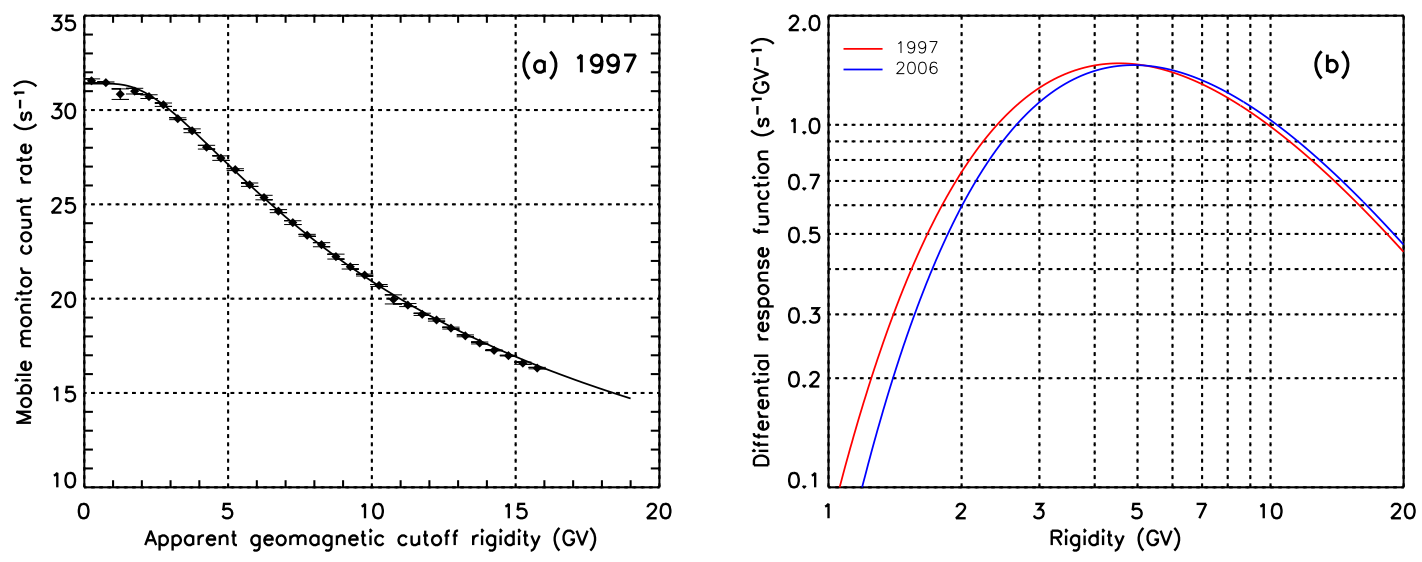

Figure 4: (a) Example of the corrected ship-borne neutron monitor count rate vs. apparent geomagnetic cutoff, for survey year 1997 (solar minimum conditions), with a Dorman function fit (solid line). (b) Differential response functions for two survey years near solar minimum, of opposite polarity and similar modulation level. A crossover is apparent near 5 GV. (Figure panels from [19].)

corrected for tube drifts or excluded. Since the mounting on the ship could be different on each voyage, we used data when the ship was near McMurdo to normalize the pressure-corrected shipborne monitor count rate based on the McMurdo NM count rate. A normalization factor was required for 5 of the 13 survey years, and was applied uniformly to all data during that survey year.

As with any neutron monitor count rate, an accurate pressure correction is vital. We corrected all data to $760 \mathrm{~mm} \mathrm{Hg}$ using an empirical pressure coefficient $\beta$ in units of percent per $\mathrm{mm} \mathrm{Hg}$ as determined from the survey data [19]:

$$
\beta=1.006-0.01534 P_{c},
$$

where $P_{c}$ is the apparent cutoff rigidity. To generate a summary set of response functions we also applied a correction for short-term variations in modulation level based on variations in the McMurdo count rate. Following [4, 8], we organized the data in terms of apparent cutoff rigidity, i.e., the effective cutoff rigidity averaged over one vertical and eight oblique directions of incidence.

\section{Results}

Figure 4(a) is an example of a response function, for the 1997 survey year. A single survey could reach the same cutoff rigidity range at up to 4 epochs while the ship traveled from Seattle to Antarctica and back (see Figure 3). We average the corrected count rates from all epochs and the error bar represents the standard error among the count rates for those epochs, which indicates the systematic (reproducibility) uncertainty. Actual statistical errors are negligible. The resulting response function $N$ for each survey year was fit to a Dorman function [11], $N=N_{0}\left(1-\exp -\alpha P_{c}^{-\kappa}\right)$. The Dorman fit parameters for all survey years are provided in [19]. We refer to $-d N / d P_{c}$ as the differential response function (DRF), and roughly speaking it represents the cosmic ray spectrum times the neutron monitor yield function. Thus the ratio between two DRFs from the same instrument, as shown in Figure 4(b) for our 1997 and 2006 survey years, directly represents the ratio 
between the cosmic ray spectra at those times. According to Figure 4(b), we observe a crossover in DRFs from successive solar minima, confirming the dependence of GCR spectral evolution on solar magnetic polarity.

Our data for 13 consecutive survey years should allow us to examine in detail the transition in spectral shape with solar magnetic polarity. However, while solar minimum is a relatively stable time with weak variability, the response functions from other survey years are generally not comparable because of different modulation levels, and cannot be expected to exhibit a literal "crossover." Instead, we believe that a startlingly simple analysis shows that the crossover is a natural consequence of a pronounced, persistent shift in the structure of modulation that occurred rather suddenly at the time of the polarity reversal in the year 2000.

Our evidence comes from a regression analysis of the ship-borne NM count rate for various cutoff rigidity ranges against the contemporaneous pressure-corrected McMurdo NM count rate. For this analysis we use the ship-borne NM count rate corrected for pressure but uncorrected for short term modulation variations, and we still exclude large Forbush decreases. Figure 5 summarizes the analysis, which consists of straight line fits to the data, divided into apparent cutoff rigidity bins of width $1 \mathrm{GV}$. To cut down on clutter, Figure 6 represents each rigidity bin for each survey by a single point at the average value for all data during that survey year, whereas the regression lines are based on the 1-hr or 30-min count rates shown in Figure 5. The regression coefficients were tabulated by [19]. Fits to a simple force-field model confirm that the latitude survey data before and after 2000 cannot be explained by the same modulation model [19].

\section{Discussion}

The crossover as typically defined has to do with spectral comparisons at "similar modulation levels." In practice, the differential response function is determined for extended time periods at yearly intervals that often do not have similar modulation levels. Our analysis shows that there is a straightforward way of avoiding this ambiguity. By comparing survey data with contemporaneous data from a fixed neutron monitor (McMurdo), we find a consistent trend with a slope that changes with solar magnetic polarity. The "crossover" is a manifestation of this basic change in modulation that occurs suddenly at the time of the solar polarity reversal. Suddenly, in this context, is defined as a year or so - which is also approximately the time it takes for the solar wind to carry the new polarity state to the outer reaches of the solar system. This sudden change of state of the heliosphere is quite like that reported for the relative abundance of protons and antiprotons [1] and electrons and positrons [7, 9]. One immediate conclusion from the observation of a rapid change in solar modulation is that we are seeing a phenomenon related to the classic solar wind, not the very distant reaches of the heliosphere at or beyond the termination shock.

There have been several direct observations of the full spectrum from balloon-borne and spacebased instruments for specific epochs. Typically measurements have not been available for the same instrument and the same level of modulation in opposite polarities, so they have not confirmed a crossover per se. The most direct comparison to the present work is provided by the series of proton spectra from the BESS payload [17] that span the year 2000 solar polarity reversal with observations from the same instrument. [17] also fit their data to a force field model which, apart from normalization, has only one adjustable parameter. Close examination reveals that the fits to 

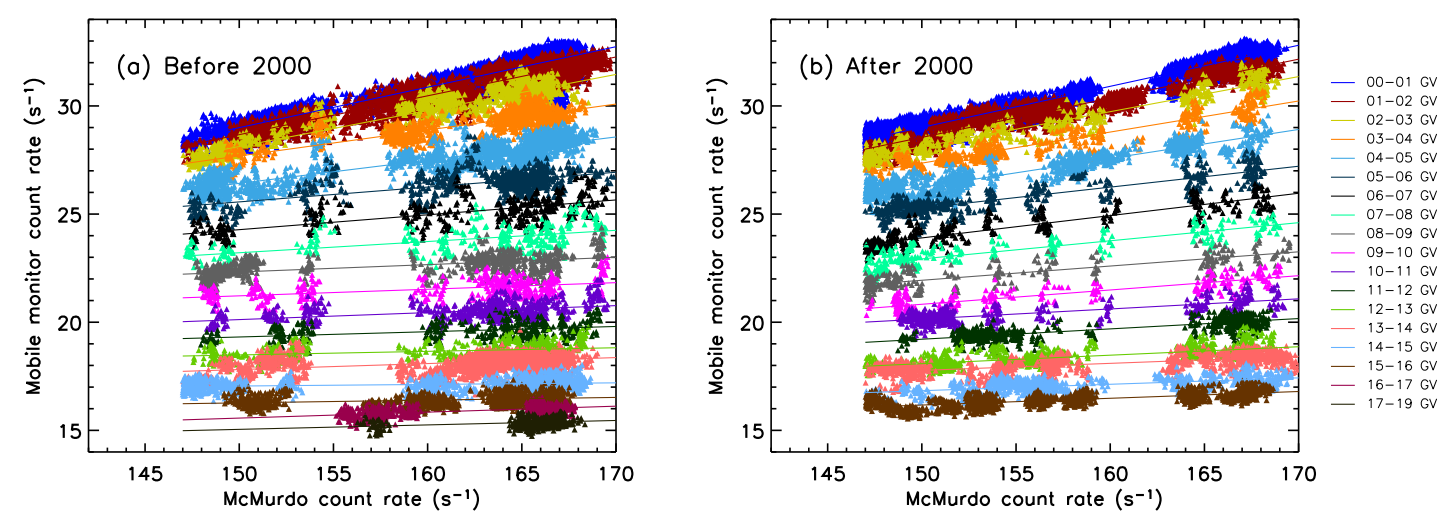

Figure 5: Scatter plot of ship-borne NM count rate vs. McMurdo NM count rate, when above $147 \mathrm{~s}^{-1}$, for all latitude surveys during (a) $A>0$ solar magnetic polarity (before 2000) and (b) $A<0$ solar magnetic polarity (after 2000). The color indicates the range of cutoff rigidity, and linear fits are shown for each range (solid lines).

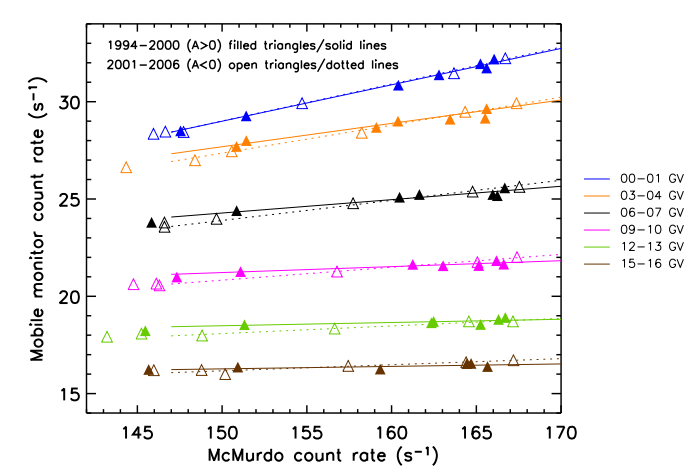

Figure 6: Alternative presentation of the data in Figure 5 using every third rigidity bin for clarity and superimposing the data for different solar magnetic polarities. Filled triangles are used to indicate positive $(A>0)$ solar magnetic polarity with solid lines showing the linear fits. Open triangles indicate data for negative $(A<0)$ solar magnetic polarity while the dotted lines are linear fits to these data. There are clear differences in cosmic ray modulation before and after the solar magnetic polarity reversal. (Figure from [19].)

spectra after the polarity reversal are not as good as those to spectra before the reversal. The fits in fact fail by overpredicting the data at low energy, just as we find in our results. We therefore consider that the BESS data are consistent with our results.

Gradient and curvature drifts are clearly established as an important factor in solar polarity dependence of modulation [14] but in and of itself the drift picture does not predict a crossover. It is not intuitively obvious why reversal of the drift fields would increase modulation at one rigidity and decrease it at another. Our observation of a rapid switch from one state to another also indicates that the tilt angle of the heliospheric current sheet is not a major factor. As [10] discuss, for observations at approximately $1 \mathrm{GV}$, the effect of current sheet tilt angle is clearly observable in precise measurements but it is tiny compared to the abrupt change with the polarity reversal itself.

Here we propose a specific mechanism to explain the different spectral evolution for different magnetic polarities. Diffusion coefficients can change radically with solar polarity because of helicity in the solar wind magnetic field and systematically organized magnetic helicity is actually observed in direct measurements of the interplanetary magnetic field. This will produce a larger mean free path during negative solar polarity, consistent with [6]. This produces a natural explana- 
tion for the crossover since the enhanced diffusion coefficients would work in the opposite direction of drifts. During the negative polarity state, when drifts operate to limit fluxes, a larger diffusion coefficient, particularly at the higher energies, permits enhanced access. With such competing effects, each having a different energy dependence, a crossover would be just an observational result that one effect dominates at low energy and the other dominates at high energy.

\section{Acknowledgments}

Partially supported by the Thailand Research Fund, United States National Science Foundation (OPP-0838839, PLR-1245939, and their predecessors), and Australian Antarctic Division.

\section{References}

[1] Asaoka, Y., Shikaze, Y., Abe, K., et al. 2002, PhRvL, 88, 051101

[2] Bieber, J. W., Burger, R. A., Engel, R., Gaisser, T. K., Roesler, S., \& Stanev, T. 1999, PhRvL, 83, 674

[3] Bieber, J. W., \& Chen, J. 1991, ApJ, 372, 301

[4] Bieber, J. W., Clem, J., \& Evenson, P. 1997, in Proc. 25th ICRC (Durban), 2, 389

[5] Carmichael, H. 1964, IQSY Instruction Manual No. 7 (London: IQSY Secretariat)

[6] Chen, J., \& Bieber, J. W. 1993, ApJ, 405, 375

[7] Clem, J., \& Evenson, P. 2004, JGR, 109, 7107

[8] Clem, J. M., Bieber, J. W., Evenson, P., Hall, D., Humble, J. E., \& Duldig, M. 1997, JGR, 102, 26919

[9] Clem, J. M., \& Evenson, P. A. 2002, ApJ, 568, 216

[10] Clem, J. M., Evenson, P., Huber, D., Pyle, R., Lopate, C., \& Simpson, J. A. 2000, JGR, 105, 23099

[11] Dorman, L. I., Fedchenko, S. G., Granitsky, L. V., \& Rishe, G. A. 1970, in Proc. 11th ICRC (Budapest), 2, 233

[12] Forbush, S. E. 1954, JGR, 59, 525

[13] Garcia-Munoz, M., Meyer, P., Pyle, K. R., Simpson, J. A., \& Evenson, P. 1986, JGR, 91, 2858

[14] Jokipii, J. R., Levy, E. H., \& Hubbard, W. B. 1977, ApJ, 213, 861

[15] Jokipii, J. R., \& Thomas, B. 1981, ApJ, 243, 1115

[16] Krüger, H., Moraal, H., Bieber, J. W., Clem, J. M., Evenson, P. A., Pyle, K. R., Duldig, M. L., \& Humble, J. E. 2008, JGR, 113, 8101

[17] Mitchell, J. W., Abe, K., Fuke, H., et al. 2008, in Proc. 30th ICRC (Mérida), 1, 455

[18] Moraal, H., Potgieter, M. S., Stoker, P. H., \& van der Walt, A. J. 1989, JGR, 94, 1459

[19] Nuntiyakul, W., Evenson, P., Ruffolo, D., Sáiz, A., Bieber, J. W., Clem, J., Pyle, R., Duldig, M. L., \& Humble, J. E. 2014, ApJ, 795, 11

[20] Oh, S., Bieber, J. W., Evenson, P., Clem, J., Yi, Y., \& Kim, Y. 2013, JGR, 118, 5431

[21] Thambyahpillai, T., \& Elliot, H. 1953, Natur, 171, 918

[22] Webber, W. R., \& Lockwood, J. A. 1988, JGR, 93, 8735 